\title{
Improving the Sensitivity of Advanced LIGO Using Noise Subtraction
}

\author{
D. Davis ${ }^{1}$, T. J. Massinger ${ }^{2}$, A. P. Lundgren ${ }^{3}$, J. C.

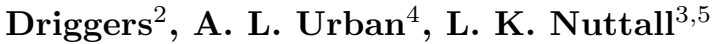 \\ ${ }^{1}$ Syracuse University, Syracuse, NY 13244, USA \\ ${ }^{2}$ California Institute of Technology, Pasadena, CA 91125, USA \\ ${ }^{3}$ University of Portsmouth, Portsmouth PO1 2UP, United Kingdom \\ ${ }^{4}$ Louisiana State University, Baton Rouge, LA 70803, USA \\ ${ }^{5}$ Cardiff University, Cardiff CF24 3AA, United Kingdom
}

\begin{abstract}
This paper presents an adaptable, parallelizable method for subtracting linearly coupled noise from Advanced LIGO data. We explain the features developed to ensure that the process is robust enough to handle the variability present in Advanced LIGO data. We demonstrate noise subtraction over the entirety of the second observing run, resulting in increases in sensitivity comparable to those reported in previous targeted efforts. Over the course of the second observing run, we see a 30\% increase in Advanced LIGO sensitivity to gravitational waves from a broad range of compact binary systems.
\end{abstract}




\section{Introduction}

Advanced LIGO's (aLIGO) second observing run (O2) lasted from November 30, 2016 to August 26, 2017. Initial analysis of the $\mathrm{O} 2$ data set resulted in the detection of gravitational-wave signals from 3 binary black hole (BBH) systems $[1,2,3]$ and the first ever detection of gravitational waves from a binary neutron star (BNS) system [4].

It has been previously shown that it is possible to increase the sensitivity of the aLIGO detectors by subtracting instrumental noise from the gravitational-wave strain data $[5,6,7,8,9]$. For source parameter estimation [10] of previously published gravitational-wave signals from O2, a MATLAB-based noise subtraction algorithm was used to subtract instrumental noise using an associated witness sensor for 4096 seconds around identified events [11, 12]. This was the first instance of noise subtraction being used in the analysis of gravitational-wave events. However, this process was not designed with the intention of subtracting noise from the entire $\mathrm{O} 2$ data set.

Since this initial analysis, a Python-based implementation of noise subtraction was developed that prioritizes parallel processing and computational efficiency with the goal of subtracting instrumental noise over the entirety of the second observing run. Considering that each individual interferometer recorded over 150 days of data, one of the key considerations was the size of the data set that this noise subtraction pipeline needed to process. The methods used in this pipeline are general enough to allow any linearly coupled noise source with a clear witness to be subtracted out efficiently.

This manuscript describes the method used to subtract noise in $\mathrm{O} 2$ and reports the improvement to search sensitivity gained by applying this method. Section 2 outlines the workflow used to process the data set in parallel. Section 3 characterizes the instrumental noise sources that were subtracted from the O2 data set. Section 4 describes the tests that were done to ensure that the subtraction process was not capable of removing genuine astrophysical signals. Section 5 presents the effects of noise subtraction on the aLIGO noise spectrum and on the sensitivity to simulated astrophysical signals.

\section{Subtraction Pipeline Overview}

\subsection{Measurement of Transfer Functions}

The assumption of a linear transfer function is motivated by the high coherence between witness sensor signals and gravitational-wave strain data. Figure 1 shows the coherence between witness sensors and gravitational-wave strain for three types of instrumental noise subtracted in $\mathrm{O} 2$. These noise sources are further detailed in Section 3.

For a given noise source, we assume that our measured gravitational wave strain data, $h(t)$, contains a noise component that can be modeled as the convolution of an unknown transfer function $c^{\prime}(t)$ and the output of a witness sensor $a(t)$,

$$
h(t)=h^{\prime}(t)+a(t) * c^{\prime}(t) .
$$

This noise component can be removed from the strain data by filtering the witness sensor data with this transfer function and subtracting its contribution to the measured strain, resulting in a residual strain denoted $h^{\prime}(t)$. This transfer function can be 
conveniently calculated in the frequency domain, so that the subtraction takes the form

$$
\tilde{h}(f)=\tilde{h}^{\prime}(f)+\tilde{a}(f) \cdot \tilde{c}^{\prime}(f) .
$$

Adapting the methodology and notation from [13], we begin by considering our data as time series that are sampled at time interval $\Delta t$ over a time period $T$. This results in $M=T / \Delta t$ samples, denoted by $Y(j)$ for $j=0, \ldots, M-1$. We denote the Discrete Fourier Transforms of each data stream as $\tilde{Y}(k)$ for $k=-M / 2, \ldots, M / 2$, so that the $k$ 'th bin corresponds to a frequency $f=k / T$. We then split the frequency space into bands of width $F$ given by

$$
f \in\left[f_{b}, f_{b+1}\right) \text { with } f_{b}=\frac{b F}{T}
$$

for $b=0, \ldots, M / 2 F$. The transfer function is measured independently over each of these frequency bands and is constructed using frequency domain inner products between the relevant data sets. For two data sets $Y_{1}$ and $Y_{2}$ the inner product over a specific frequency band $b$ is calculated as the cross-power spectrum summed over that frequency band:

$$
\tilde{c}_{12}\left(f_{b}\right)=\sum_{f=f_{b}}^{f_{(b+1)}} \tilde{Y}_{1}(f) \tilde{Y}_{2}{ }^{*}(f) .
$$

A measurement of the transfer function for uncorrelated noise, for which each frequency bin has a random phase, should find no significant coupling as multiple uncorrelated data points are averaged over to calculate the transfer function. To help reduce the risk of spurious correlations being measured, we set a minimum threshold on the value that the transfer function can take as a fraction of the maximum value and set the value of the transfer function to zero in any band whose value is below that threshold.

In the case when multiple sensors witness the same noise, there will be a measurable correlation between each of the sensors, resulting in oversubtraction if not accounted for. For $N$ witness sensors $Y_{1}, \ldots Y_{N}$ and a target data stream to subtract noise from, $Y_{0}$, the set of frequency domain transfer functions $\tilde{c}_{01}^{\prime}, \ldots \tilde{c}_{0 N}^{\prime}$ that contain independent noise is the solution to the matrix equation [13]

$$
\left[\begin{array}{c}
\tilde{c}_{01}^{\prime}\left(f_{b}\right) \\
\tilde{c}_{02}^{\prime}\left(f_{b}\right) \\
\vdots \\
\tilde{c}_{0 N}^{\prime}\left(f_{b}\right)
\end{array}\right]=\left[\begin{array}{ccc}
\tilde{c}_{11}\left(f_{b}\right) & \ldots & \tilde{c}_{N 3}\left(f_{b}\right) \\
\tilde{c}_{12}\left(f_{b}\right) & \ldots & \tilde{c}_{N 2}\left(f_{b}\right) \\
\vdots & \ddots & \vdots \\
\tilde{c}_{1 N}\left(f_{b}\right) & \ldots & \tilde{c}_{N N}\left(f_{b}\right)
\end{array}\right]^{-1}\left[\begin{array}{c}
\tilde{c}_{01}\left(f_{b}\right) \\
\tilde{c}_{02}\left(f_{b}\right) \\
\vdots \\
\tilde{c}_{0 N}\left(f_{b}\right)
\end{array}\right]
$$

These independent transfer functions can then be used for noise subtraction as described in Equation 2. This process allows additional sensors that may witness different features of the same noise source to be added to the noise subtraction process without risking oversubtraction.

\subsection{Calculation of Coupled Noise}

Advanced LIGO data is not stationary on the time scale of hours [14, 15], meaning the transfer functions used to subtract each noise source will vary over the time period 


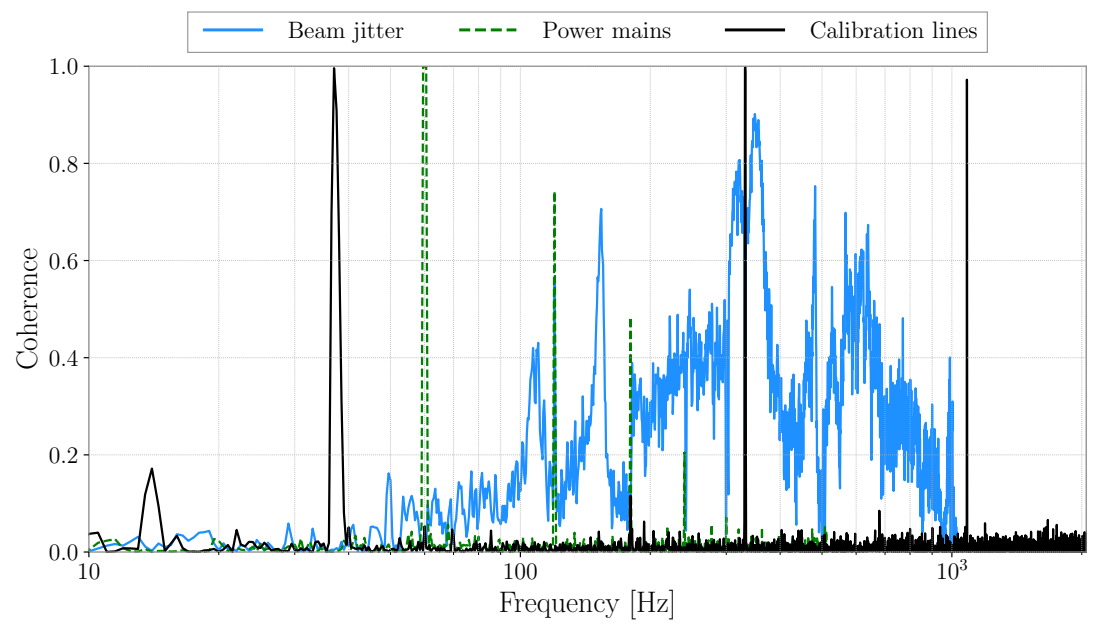

Figure 1: Coherence between witness sensors and gravitational wave strain for three types of instrumental noise subtracted in $\mathrm{O} 2$ at Hanford: beam jitter, power mains, and calibration lines. The measured coherence demonstrates significant linear coupling between these witness sensors and the strain data, motivating the use of linear subtraction methods.

that the noise subtraction is applied. This necessitated the development of methods to understand the stability and accuracy of the transfer function estimation on long timescales.

High amplitude non-Gaussian instrumental artifacts that can impact the measurement of transfer functions are removed from the strain data before transfer functions are calculated. This is done by applying an inverse Tukey window that zeroes the data containing each instrumental transient. This process is identical to the windowing done in [4]. A continuous measurement for long stretches of data is approximated by calculating transfer functions with overlapping finite measurement windows, called "sections." A visualization of this process is shown in Figure 2. After calculating the transfer functions and projected noise contributions for each individual window, each section of projected noise is multiplied by a Hann window and smoothly added together with overlapping sections.

In the case that the transfer function is truly constant, this method is identical to applying a single transfer function over the entire period. The transfer function for each witness sensor is constructed to be uncorrelated with the transfer functions from other witness sensors, resulting in noise projection time series that are also independent. This allows each noise time series to be subtracted from the strain data independently. Once all targeted noise contributions are subtracted, we refer to the data as "cleaned".

\subsection{Workflow Implementation}

One of the key features of this implementation is the throughput at which the subtraction can be done over long stretches of data. The pipeline takes advantage of the Pegasus workflow methods implemented in the PyCBC software package 


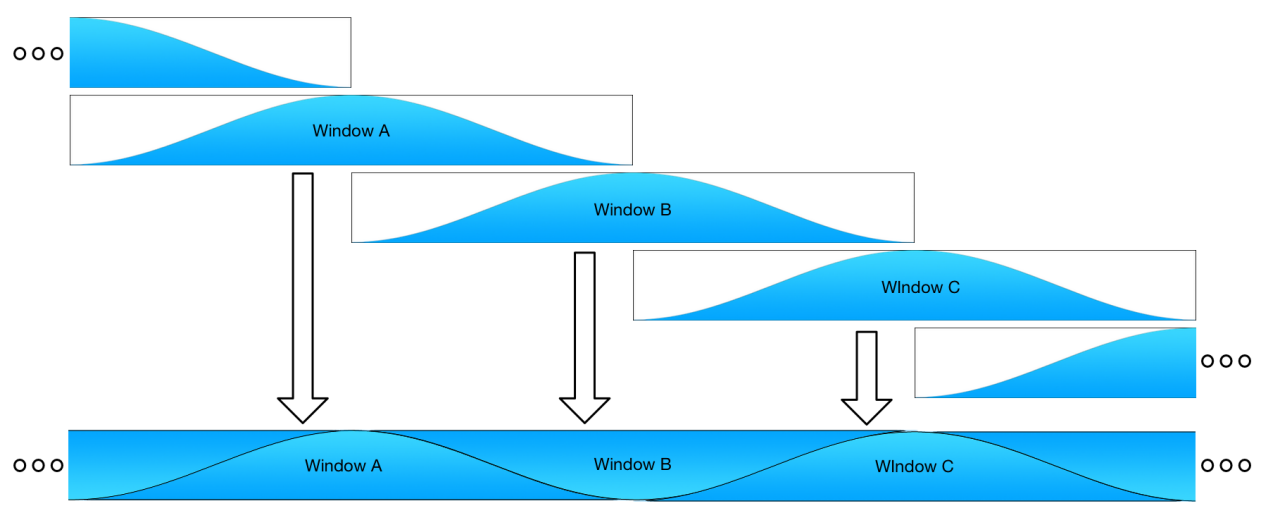

Figure 2: Visualization of how transfer function measurements are tiled in time. Transfer functions are measured in time windows (typically 1024 seconds) with $50 \%$ overlap. For a given time, the transfer function between the witness sensor and $h(t)$ is measured and the witness data are filtered to generate their projected contributions to $h(t)$. A Hann window is applied to each section of projected data before adding them together, resulting in a single projected $h(t)$ time series that has incorporated the time dependence of the transfer functions.

$[16,17,18]$, which allows for parallelized calculation of transfer functions. Since the transfer functions for different noise sources can be calculated independently, the workflow was able to measure transfer functions for each noise source and generate projected strain data in parallel. In addition, the data set was broken up into distinct sections of continuous detector operation that were processed in parallel. The limiting factor in the subtraction process is the availability of computing nodes. Applying this method using available resources with 14 witness sensors allowed for two weeks of data from one detector, approximately 65 gigabytes, to be processed in only a few hours.

\section{Noise sources}

During O2, multiple sources of linearly coupled noise were identified. These fell into two main categories: beam jitter noise that led to broadband noise contributions and narrow line artifacts from power mains and calibration lines. Both of these noise classes were identified and subtracted for analyses on previously published events, as described in [11]. This section describes each noise source and the witness sensors used in the subtraction process.

\subsection{Jitter Noise}

The main source of linearly coupled noise identified during $\mathrm{O} 2$ was related to jitter of the pre-stabilized laser (PSL) beam in angle and size [19]. The PSL is responsible for generating the frequency- and intensity-stabilized input laser beam that is injected into the interferometer. Upgrades to this subsystem undertaken in preparation for O2 led to different configurations of the PSL between Hanford and Livingston.

The configuration of the PSL at Hanford during O2 included the addition of a 
high powered oscillator (HPO) that was designed to increase the laser power injected into the interferometer up to $200 \mathrm{~W}[19,20]$. The optical components used in the HPO required continuous heat dissipation via water cooling. Vibrations from water flow coupled to the table that supports the optical components used to control the beam angle, introducing jitter in beam angle and size [21].

Fluctuations in beam angle are measured using quadrant photodiodes that sense the light reflected from the input mode cleaner (IMC) [22], which is used to filter higher order optical modes from the input beam. In February 2017, an additional sensor sensitive to radial beam distortions was installed [23].

During O2, the coupling of beam jitter into the output of the detector was further complicated by the presence of an axially asymmetric point absorber that was present on one of the test masses at Hanford [24]. Thermal deformations are generally corrected with the use of the the Thermal Compensation System (TCS), which heats and deforms the mirrors [25], but this system is not capable of compensating for a pointlike deformation. This deformation may have caused beam size and angle fluctuations to more strongly couple into the gravitational-wave strain data.

Jitter noise related to beam size and beam angle fluctuations was present at Hanford throughout all of $\mathrm{O} 2$, with increased coupling towards the end of the run. Variations in the beam angle size led to broadband noise contributions, while variation in beam angle was coupled strongly at mechanical resonances of optic mounts between 100 and $700 \mathrm{~Hz}$. The sensors used to witness these noise sources were digitally sampled at $2048 \mathrm{~Hz}$, which sets the maximum frequency at which this jitter noise can be subtracted at $1024 \mathrm{~Hz}$. The broadband coupling may have introduced noise above this frequency, but is not addressed in this work.

At Livingston, the HPO was not included in the $\mathrm{O} 2$ configuration, and no asymmetries in the test masses were noted, leading to no noticeable jitter coupling in the gravitational-wave strain data. This accounts for the lack of broadband noise subtraction seen in the Livingston spectrum shown in Figure 4.

\subsection{Line Artifacts}

The gravitational-wave strain data demonstrates several noise features that are narrowband, appearing as sharp lines in the frequency domain that can affect long duration searches and parameter estimation. The strain data contains excess noise at $60 \mathrm{~Hz}$ and its harmonic frequencies at both sites due to coupling of the power mains. These lines can be subtracted out using witness sensors that directly measure the 3 -phase voltage provided by the mains power grid at each observatory. In addition, the calibration lines discussed in Section 4.4 are applied using two methods. One set of calibration lines are digitally injected into actuation signals that control the position of the optics. These injected signals can be measured directly and subtracted using readouts at the injection points. A second set of calibration lines are applied to the test masses via radiation pressure using the photon calibrator [26] and can be measured and subtracted using a photodetector that monitors the power of the photon calibrator beam. 


\section{Diagnostics}

\subsection{Sensor Safety}

Before using the witness sensors described in Section 3 to subtract correlated noise, each sensor's sensitivity to gravitational waves, or "safety", was estimated. To establish safety, a series of sine-Gaussian waveforms were injected into the detector to excite the degree of freedom that is sensitive to gravitational waves [27]. If an excitation of this degree of freedom coupled into the readout of any witness sensors in a statistically significant way [28], those sensors were considered capable of accidentally subtracting away real gravitational-wave signals and were marked as unsafe. All of the witness sensors used for noise subtraction were determined to be incapable of witnessing and subtracting away gravitational-wave signals.

\subsection{Recovery of Simulated Compact Binary Coalescence Signals}

To ensure the noise subtraction process would not corrupt an astrophysical signal, a set of simulated compact binary coalescence (CBC) waveforms was digitally inserted over five days worth of aLIGO data from both detectors. The data set containing these simulated signals was processed by the PyCBC astrophysical search algorithm $[17,18]$ before and after subtracting noise, and the recovery of the simulated signals was compared. For each recovered signal, a coincident ranking statistic that represents the significance of an event found in multiple detectors in the detector network is calculated. Figure 3 shows the recovered coincident ranking statistic of each simulated signal before and after subtracting noise from the data set. After noise subtraction, all of the simulated signals were recovered with a ranking statistic that is consistent with or better than the ranking statistic before subtraction. In addition, there is a population of simulated signals that were not recovered in the original analysis but were found as coincident events after noise subtraction. As a final test, hardware injected CBC signals [27] were successfully recovered after performing noise subtraction. These hardware injections were recovered with increases in ranking statistic consistent with changes seen in software injections.

\subsection{Simulated Noise Tests}

To verify that the noise subtraction process is effective for generic noise sources, artificial noise was added to aLIGO strain data and processed using the same method. The first test attempted to subtract artificial correlated noise. This noise was constructed by generating Gaussian noise, passing it through a transfer function that had similar features to the jitter transfer function, and summing it into the strain data. When provided with the strain data and the Gaussian noise, the noise subtraction algorithm was able to reconstruct the transfer function used to project the Gaussian noise into the strain data and subtract out the excess noise. The amplitude spectral density of the resulting data was consistent with the original data to within $\pm 3 \%$ at all frequencies.

The second test was to subtract out random, uncorrelated noise which had not been added to the strain data. When provided with the strain data and the uncorrelated Gaussian noise, the algorithm subtracted a minimal amount of random

noise. Similarly, the amplitude spectral density of the resulting data was consistent with the original data to within $\pm 2 \%$ at all frequencies. 


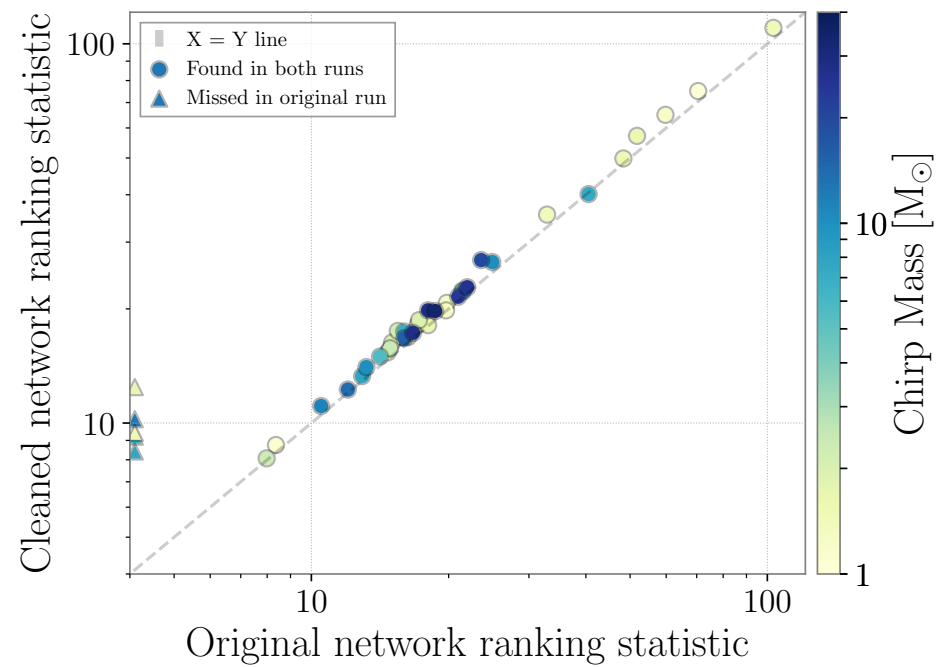

Figure 3: Recovered network ranking statistic for simulated gravitational wave signals before and after applying noise subtraction. The colorbar indicates the chirp mass [29] of each event, which spans a large astrophysical parameter space including binary neutron Star, neutron star - black Hole, and binary black hole signals. After noise subtraction, the simulated signals are recovered with a network ranking statistic that is greater than or equal to the ranking statistic without noise subtraction. In addition, several quiet simulated signals that were below the threshold of the search pipeline were recovered after noise subtraction due to being below the minimum threshold of signal to noise ratio of 5.5 in both detectors. These are indicated with triangles.

\subsection{Effect on Calibration}

One important feature of aLIGO data is the presence of continuous, narrowband sinusoidal injections, or "calibration lines", which are used to calibrate the data [30]. This calibration is performed on data that does not have noise subtracted, therefore tests were conducted to ensure that the calibration of the data was still valid after cleaning. A set of noise subtracted data was produced using data from Hanford that did not subtract away calibration lines in order to measure the impact of broadband noise subtraction on the data calibration process. Both the cleaned and uncleaned strain data were demodulated at the calibration line frequencies and the amplitude and phase were averaged in 300 second bins. The amplitude ratio and phase offset of each resulting measurement were calculated and are used as metrics for consistency.

To accumulate a statistically significant measurement of the calibration line consistency, 6.65 days of data were analyzed and the $1 \sigma$ errors on the distribution of amplitude ratios and phase offsets are reported. For the $36.7 \mathrm{~Hz}$ and $1083.7 \mathrm{~Hz}$ calibration lines at Hanford, the amplitude ratio was consistent with 1 to within $\pm 0.014 \%$ and the phase offset was consistent with $0^{\circ}$ to within $\pm 0.0078^{\circ}$. The 331 $\mathrm{Hz}$ calibration line (Located at a frequency where a non-negligible amount of power is expected to be subtracted off due to beam jitter) has an amplitude ratio that is consistent with 1 to within $\pm 0.15 \%$ and a phase offset that is consistent with $0^{\circ}$ to 
within $\pm 0.087^{\circ}$. As typical calibration uncertainties are $\pm 4 \%$ [31], these measurements confirm that the noise subtraction process did not significantly impact the overall calibration of the strain data.

\subsection{Impact of Nonstationary Data}

While generally stable, the witness sensors used for transfer function estimation sometimes contain transient noise. In cases where the witness sensor has transient excess power that is not linearly correlated to the gravitational-wave strain, the transfer function is overestimated and the noise subtraction algorithm removes too much projected noise from the strain data. However, when the transient noise is linearly correlated to the gravitational-wave strain, transient noise can be subtracted from the gravitational-wave strain data. This linear subtraction of transient noise was commonly found during periods of transient noise in the power mains.

The most impactful cases of oversubtraction due to excess power that is not linearly correlated with the gravitational-wave strain were noticed during review of the cleaned data set, and occur when there is transient noise in the photon calibrator used to inject calibration lines into the detector. To avoid this overestimation, the noise subtraction process is halted for 3 seconds around these transient noise artifacts. Once times where witness sensors contain transient noise are removed, the nearby noise subtracted data shows no evidence of oversubtraction.

Additional oversubtraction may occur if a feature of a witness sensor is spuriously correlated with the gravitational wave data. While such features are not observed on the timescales that the subtraction process is computed over, narrowband noise features from beat notes in the photon calibrator system may appear when signals are averaged on the timescale of multiple hours. The total bandwidth affected by these spurious correlations is less than $0.1 \mathrm{~Hz}$ and can be removed from long timescale analyses with the use of notch filters [32].

\section{Results}

\subsection{The O2 Data Set}

The noise subtraction algorithm was used to clean the entire data set from Advanced LIGO's second observing run, which spanned 9 months. The final version of calibrated data $[30,31]$ was used as the input to the noise subtraction pipeline. For computational efficiency, data which were considered unfit for astrophysical analysis [14, 33, 34, 35] were not processed. Additional time losses were due to removal of time corrupted by bandpass filters applied in the subtraction process and the excision of data where witness sensors were unsuitable for reliable transfer function measurement, as noted in Section 4.5. In all, only $0.05 \%$ of strain data was discarded as a result of the noise subtraction process. The final cleaned data set contains 118 days of coincident data. This value is greater than the coincident livetime reported in [4] due to the inclusion of additional time with updated calibration [31, 30].

\subsection{Effects on the Noise Curve}

The noise subtraction process is capable of removing both narrowband and broadband spectral features. Figure 4 shows the amplitude spectral density of the strain data from the Hanford detector before and after noise subtraction. The narrow lines removed 

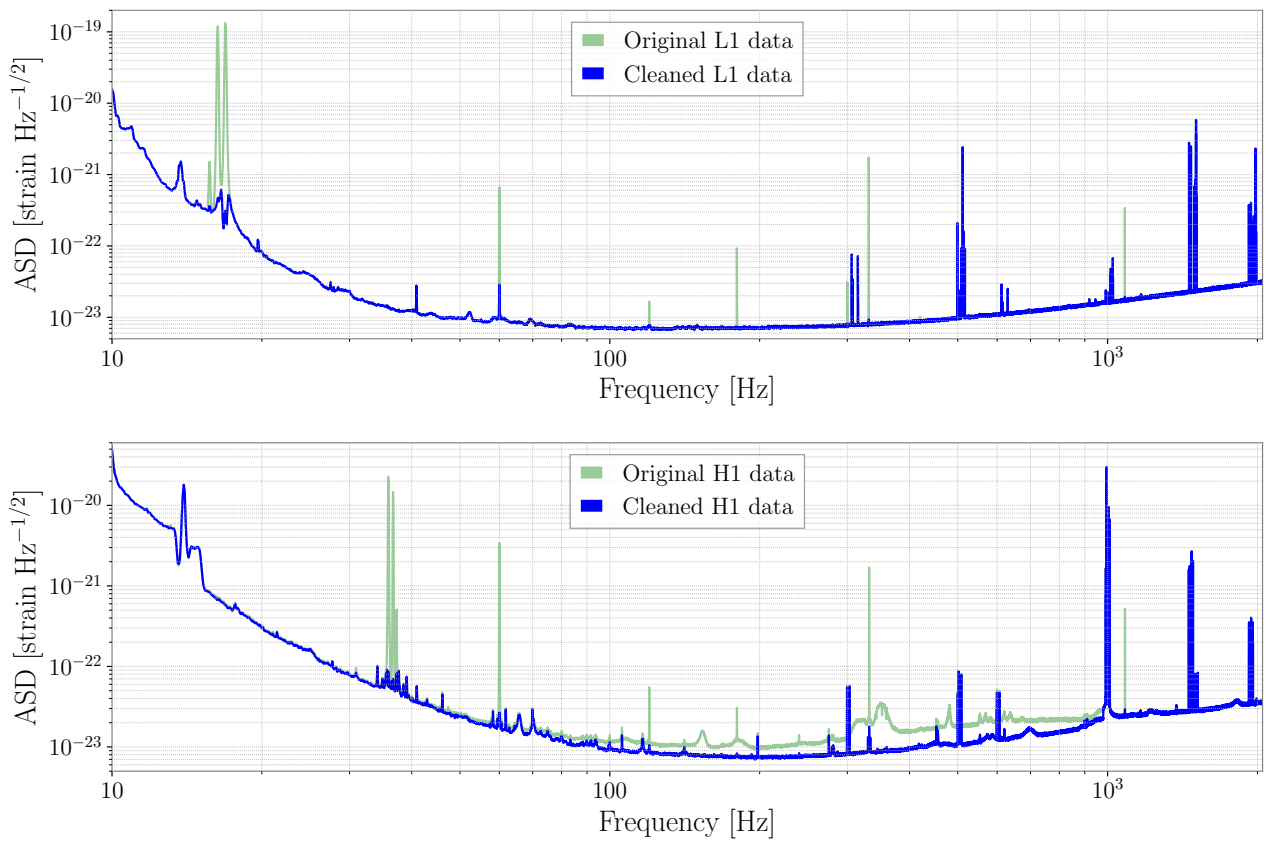

Figure 4: Top: Amplitude spectral density (ASD) of Livingston (L1) gravitational wave strain data before (green) and after (blue) noise subtraction from a representative day of data during O2. Narrowband features from calibration lines and power mains were subtracted from the L1 strain data. There were no broadband noise sources with an appropriate witness sensor that could be subtracted from the L1 strain data. Bottom: Amplitude spectral density of Hanford (H1) gravitational wave strain data before and after noise subtraction from a representative day of data during O2. In addition to narrowband features from calibration lines and power mains, broadband noise was subtracted between 80 - $1000 \mathrm{~Hz}$ using beam jitter witness sensors.

at $33,60,120,180,331$, and $1083 \mathrm{~Hz}$, detailed in Section 3.2, are related to detector calibration lines and power mains harmonics. Broadband subtraction in Hanford data is a result of removing noise related to beam jitter. Due to the $2048 \mathrm{~Hz}$ sampling rate of the witness sensors and a low pass filter applied to reduce corruption near the Nyquist frequency, broadband noise is only subtracted up to $1024 \mathrm{~Hz}$. An additional high pass filter applied at $13 \mathrm{~Hz}$ set the minimum frequency broadband noise was subtracted.

The same procedure was used to address noise sources present in the Livingston detector. Line artifacts due to calibration lines and harmonics of the power mains were removed. As previously noted, beam jitter noise did not contribute significantly to the Livingston data and was not subtracted.

We can characterize the benefit of the noise subtraction process with the "inspiral range", which is the average distance at which a detector could observe a BNS system $\left(1.4-1.4 \mathrm{M}_{\odot}\right)$ at a signal to noise ratio (SNR) of 8 . The inspiral range during $\mathrm{O} 2$ at Hanford before and after noise subtraction is shown in Figure 5. After noise subtraction, the inspiral range at Hanford increased by $\sim 20 \%$ when averaged over 


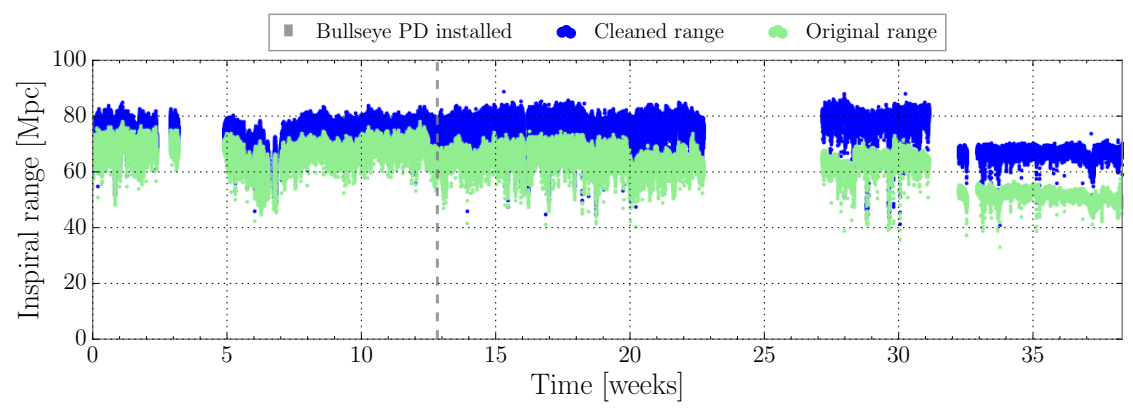

Figure 5: Inspiral range of the Hanford (H1) detector over the course of O2 before (green) and after (blue) noise subtraction. The dashed line indicates the installation of the bullseye photodiode, a witness sensor used for subtraction of noise due to beam jitter. The large decrease in range after week 32 for both the Original and Cleaned range was due to the impact of an earthquake near the site [36]. Livingston (L1) had no broadband noise subtraction, the increase in inspiral range was negligible and is not shown.

all of $\mathrm{O} 2$ with a peak increase of $\sim 50 \%$ towards the end of the observing run. The change in inspiral range at Livingston was negligible over the course of $\mathrm{O} 2$ due to the lack of broadband noise subtraction. The effect of noise subtraction on the overall network sensitivity of the detectors is discussed in Section 5.3.

\subsection{Effect on Astrophysical Analyses}

The figure of merit used for quantifying sensitivity of a search to astrophysical signals is the sensitive volume of the search multiplied by the analysis time, which is known as volume-time (V-T). While this volume can be approximated using the inspiral range as a measure of sensitive distance, that method does not fully account for the effects of data containing non-Gaussian noise artifacts on astrophysical search sensitivity, as well as the sensitivity of the entire interferometer network. V-T can be measured by injecting an population of simulated gravitational-wave signals into the data and attempting to recover them with a search pipeline [17]. For each search pipeline, a background distribution is generated that excludes coincident events in order to estimate the effects of detector noise on the search algorithm. Each recovered signal is then compared to this background and assigned an inverse false alarm rate (IFAR) that quantifies how likely it is that such a signal was caused by coincident instrumental artifacts rather than an astrophysical source. To estimate the increase in sensitivity due to noise subtraction, V-T of the PyCBC search was measured before and after noise subtraction using identical injection sets. As multiple values can be used as a cutoff to determine if a signal is recovered, we examined the $\mathrm{V}$ - $\mathrm{T}$ for IFAR values of both 100 years and 1000 years. The ratio of V-T before and after noise subtraction binned by chirp mass is shown in Figure 6 .

The large increase to the sensitive volume of the detector network, combined with a negligible reduction in available coincident time, led to a significant increase in $\mathrm{V}-\mathrm{T}$ over the course of $\mathrm{O} 2$. Averaging over all mass bins, a $30 \%$ increase in V-T was measured over the course of $\mathrm{O} 2$. Particularly, the largest gains in sensitivity were realized for chirp mass between $1.74 \mathrm{M}_{\odot}$ and $8.07 \mathrm{M}_{\odot}$. This is a parameter space that 


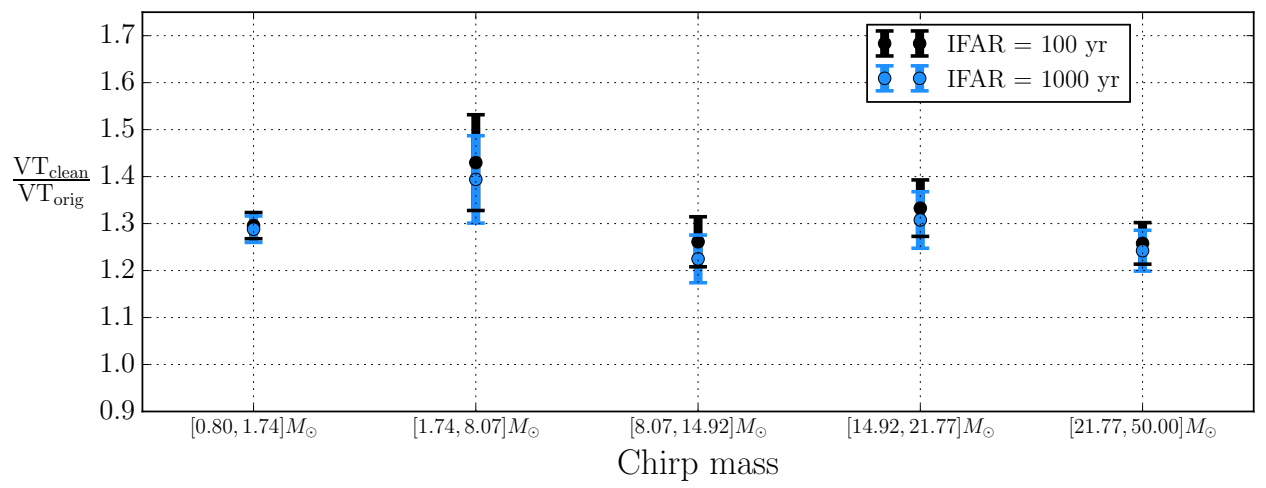

Figure 6: The ratio of volume-time (V-T) the PyCBC search was sensitive to during $\mathrm{O} 2$ before (original) and after noise subtraction (clean) binned by chirp mass. Black represents the volume-time that the search was sensitive to for signals with an inverse false alarm rate (IFAR) of 100 years, while Blue corresponds to signals with an IFAR of 1000 years. Error bars show 1 sigma error. On average, a $30 \%$ increase in $\mathrm{V}-\mathrm{T}$ was measured over the course of $\mathrm{O} 2$.

aLIGO has not previously detected signals in, and hence has a largely unconstrained rate, in addition to being the location of the observed NS-BH mass gap [37, 38, 39].

One observed effect that led to a difference in measured V-T versus V-T extrapolated from the cubing the increase in inspiral range was the impact of the noise subtraction process on instrumental artifacts. While the noise subtraction process reduced the broadband noise in the detector, it did not affect the amplitude of noise artifacts unrelated to the noise sources addressed by the noise subtraction pipeline. With a lower noise floor and no change in their absolute amplitude, artifacts already present in the data were found to increase in SNR. As one of the primary limitations of an astrophysical search's ability to recover signals is the rate of loud noise artifacts [14], these SNR increases limit the increase in V-T due to noise subtraction.

\section{Future prospects}

This paper presents an adaptable, parallelized method for subtracting linearly coupled noise from aLIGO data. The versatility of this method to remove known sources of noise makes it a useful tool to address a variety of noise sources in future observing runs. While beam jitter and line artifacts are the only noise sources subtracted from this data set, noise from feedback loops used to sense and control the length and alignment of optical cavities in the aLIGO detectors have been shown to contribute noise at lower frequencies [11] and are potential candidates for subtraction in future observing runs. Before the aLIGO's third observing run, the test mass with the point absorber will be replaced and the PSL configuration will be updated, which are expected to reduce noise contributions due to beam jitter.

The sensitivity gains demonstrated in this paper show that a robust offline noise subtraction pipeline is an integral aspect of achieving maximum sensitivity in gravitational-wave detectors. The $30 \%$ increase in sensitivity of aLIGO to compact binary coalescences after noise subtraction will allow for an increased volume of 
spacetime to be searched for gravitational waves. Although not quantified in this work, the noise subtraction process will also lead to general increases in the sensitivity of searches for gravitational waves using aLIGO data, such as those for continuous waves [40], stochastic [41], and unmodeled burst sources [42, 43, 44].

\section{Acknowledgments}

We would like to thank the aLIGO commissioners and the Detector Characterization group for identifying and characterizing the noise sources addressed, as well as the PyCBC search group for development of the injection sets used in this work. We also thank review team members Gabriele Vajente and Francesco Salemi for helpful discussions during development, along with Jess McIver and Marissa Walker for their comments during the during the internal review process. Computing support for this project was provided by the LDAS computing cluster at the California Institute of Technology. DD acknowledges support from NSF award PHY-1607169. LKN received funding from the European Union Horizon 2020 research and innovation programme under the Marie Sklodowska-Curie grant agreement No 663830. TJM acknowledges support from the LIGO Laboratory. LIGO was constructed by the California Institute of Technology and Massachusetts Institute of Technology with funding from the National Science Foundation, and operates under cooperative agreement PHY0757058. This paper carries LIGO Document Number ligo-p1800169.

The authors thank the LIGO Scientific Collaboration for access to the data and gratefully acknowledge the support of the United States National Science Foundation (NSF) for the construction and operation of the LIGO Laboratory and Advanced LIGO as well as the Science and Technology Facilities Council (STFC) of the United Kingdom, and the Max-Planck-Society (MPS) for support of the construction of Advanced LIGO. Additional support for Advanced LIGO was provided by the Australian Research Council.

\section{References}

[1] B P Abbott et al. GW170104: Observation of a 50-Solar-Mass Binary Black Hole Coalescence at Redshift 0.2. Phys. Rev. Lett., 118:221101, 2017.

[2] B P Abbott et al. GW170608: Observation of a 19 Solar-mass Binary Black Hole Coalescence. The Astrophysical Journal Letters, 851(2):L35, 2017.

[3] B P Abbott et al. GW170814: A Three-Detector Observation of Gravitational Waves from a Binary Black Hole Coalescence. Phys. Rev. Lett., 119:141101, 2017.

[4] B P Abbott et al. GW170817: Observation of Gravitational Waves from a Binary Neutron Star Inspiral. Phys. Rev. Lett., 119:161101, 2017.

[5] J C Driggers, M Evans, K Pepper, and R X Adhikari. Active noise cancellation in a suspended interferometer. Review of Scientific Instruments, 83(2):024501, 2012.

[6] R DeRosa, J C Driggers, D Atkinson, H Miao, V Frolov, M Landry, J A Giaime, and R X Adhikari. Global feed-forward vibration isolation in a km scale interferometer. Classical and Quantum Gravity, 29(21):215008, 2012.

[7] V Tiwari, M Drago, V Frolov, S Klimenko, G Mitselmakher, V Necula, G Prodi, V Re, F Salemi, G Vedovato, and I Yakushin. Regression of environmental noise in LIGO data. Classical and Quantum Gravity, 32(16):165014, 2015.

[8] G D Meadors, K Kawabe, and K Riles. Increasing LIGO sensitivity by feedforward subtraction of auxiliary length control noise. Classical and Quantum Gravity, 31(10):105014, 2014.

[9] M Coughlin, N Mukund, J Harms, J Driggers, R Adhikari, and S Mitra. Towards a first design of a Newtonian-noise cancellation system for Advanced LIGO. Classical and Quantum Gravity, $33(24): 244001,2016$ 
[10] J Veitch et al. Parameter estimation for compact binaries with ground-based gravitational-wave observations using the LALInference software library. Phys. Rev. D, 91:042003, Feb 2015.

[11] J C Driggers et al. Offline noise subtraction for Advanced LIGO. Technical document LIGOP1700260, 2017.

[12] J C Driggers et al. Improving astrophysical parameter estimation via offline noise subtraction for Advanced LIGO. 2018. arXiv:1806.00532.

[13] B. Allen et al. Observational limit on gravitational waves from binary neutron stars in the galaxy. 83:1498, 1999.

[14] B P Abbott et al. Effects of data quality vetoes on a search for compact binary coalescences in Advanced LIGO's first observing run. Classical and Quantum Gravity, 35(6):065010, 2018.

[15] M. Walker, Agnew, A.F., J. Bidler, A. Lundgren, A. Macedo, D. Macleod, O. Patane, Massinger, T. J., and Smith, J.R. Identifying correlations between LIGO's astronomical range and auxiliary sensors using lasso regression. 2018. arXiv:1807.02592.

[16] E Deelman et al. Pegasus: a workflow management system for science automation. Future Generation Computer Systems, 46:17-35, 2015. Funding Acknowledgements: NSF ACI SDCI 0722019, NSF ACI SI2-SSI 1148515 and NSF OCI-1053575.

[17] S A Usman et al. An improved pipeline to search for gravitational waves from compact binary coalescence. 2015.

[18] A H Nitz et al. PyCBC Software. https://github.com/ligo-cbc/pycbc, 2017.

[19] P Kwee et al. Stabilized high-power laser system for the gravitational wave detector advanced LIGO. Opt. Express, 20(10):10617-10634, May 2012.

[20] J Aasi et al. Advanced ligo. Classical and Quantum Gravity, 32(7):074001, 2015.

[21] R Schofield. aLIGO LHO Logbook. https://alog.ligo-wa.caltech.edu/aLOG/index.php? callRep=30290.

[22] G Vajente. aLIGO LHO Logbook. https://alog.ligo-wa.caltech.edu/aLOG/index.php? callRep=302734.

[23] S Dwyer. aLIGO LHO Logbook. https://alog.ligo-wa.caltech.edu/aLOG/index.php? callRep=34154.

[24] A Brooks. aLIGO LHO Logbook. https://alog.ligo-wa.caltech.edu/aLOG/index.php? callRep=34853.

[25] A F Brooks et al. Overview of Advanced LIGO adaptive optics. Appl. Opt., 55(29):8256-8265, Oct 2016.

[26] S Karki et al. The Advanced LIGO photon calibrators. Review of Scientific Instruments, $87: 114503,2016$.

[27] C Biwer et al. Validating gravitational-wave detections: The Advanced LIGO hardware injection system. Phys. Rev. D, 95:062002, Mar 2017.

[28] J R Smith, T Abbott, E Hirose, N Leroy, D MacLeod, J McIver, P Saulson, and P Shawhan. A hierarchical method for vetoing noise transients in gravitational-wave detectors. Classical and Quantum Gravity, 28(23):235005, 2011.

[29] C Cutler and E Flanagan. Gravitational waves from merging compact binaries: How accurately can one extract the binary's parameters from the inspiral waveform? Phys. Rev. D, 49:2658, 1994.

[30] A D Viets et al. Reconstructing the calibrated strain signal in the Advanced LIGO detectors. Classical and Quantum Gravity, 35(9):095015, 2018.

[31] C Cahillane et al. Calibration uncertainty for Advanced LIGO's first and second observing runs. Phys. Rev. D, 96:102001, Nov 2017.

[32] P B Covas et al. Identification and mitigation of narrow spectral artifacts that degrade searches for persistent gravitational waves in the first two observing runs of Advanced LIGO. Phys. Rev. D, 97:082002, 2018.

[33] L K Nuttall et al. Improving the Data Quality of Advanced LIGO Based on Early Engineering Run Results. Class. Quantum Grav., 32(24):245005, 2015.

[34] L. K. Nuttall. Characterizing transient noise in the ligo detectors. Philosophical Transactions of the Royal Society of London A: Mathematical, Physical and Engineering Sciences, 376(2120), 2018.

[35] Beverly K. Berger. Identification and mitigation of advanced ligo noise sources. Journal of Physics: Conference Series, 957(1):012004, 2018.

[36] J Kissel et al. aLIGO LHO Logbook. https://alog.ligo-wa.caltech.edu/aLOG/index.php? callRep=37846.

[37] Tyson B Littenberg, Ben Farr, Scott Coughlin, Vicky Kalogera, and Daniel E Holz. Neutron stars versus black holes: Probing the mass gap with ligo/virgo. The Astrophysical Journal Letters, 807(2):L24, 2015. 
[38] Will M Farr, Niharika Sravan, Andrew Cantrell, Laura Kreidberg, Charles D Bailyn, Ilya Mandel, and Vicky Kalogera. The mass distribution of stellar-mass black holes. The Astrophysical Journal, 741(2):103, 2011.

[39] Feryal zel, Dimitrios Psaltis, Ramesh Narayan, and Jeffrey E. McClintock. The black hole mass distribution in the galaxy. The Astrophysical Journal, 725(2):1918, 2010.

[40] K Riles. Recent searches for continuous gravitational waves. Phys. Rev. A, 32:1730035, 2017.

[41] $\mathrm{T}$ Regimbau. The astrophysical gravitational wave stochastic background. Research in Astronomy and Astrophysics, 11(4):369, 2011.

[42] R Lynch, S Vitale, R Essick, E Katsavounidis, and F Robinet. An information-theoretic approach to the gravitational-wave burst detection problem. 2015.

[43] S. Klimenko et al. Coherent method for detection of gravitational wave bursts. Class. Quantum Grav., 25:114029, 2008.

[44] N J Cornish and T B Littenberg. BayesWave: Bayesian Inference for Gravitational Wave Bursts and Instrument Glitches. Class. Quant. Grav., 32(13):135012, 2015. 